\title{
The common PPAR- $\gamma 2$ Pro12Ala variant is associated with greater insulin sensitivity
}

\author{
Raffaella Buzzetti ${ }^{* 1}$, Antonio Petrone ${ }^{1,3}$, Maria Cristina Ribaudo ${ }^{1}$, Irene Alemanno ${ }^{1}$, \\ Sara Zavarella ${ }^{1}$, Charles A Mein ${ }^{3}$, Francesca Maiani ${ }^{1}$, Claudio Tiberti $^{1}$, \\ Marco Giorgio Baroni ${ }^{1}$, Elio Vecci ${ }^{1}$, Marcello Arca ${ }^{2}$, Frida Leonetti ${ }^{1}$ and \\ Umberto Di Mario ${ }^{1}$ \\ ${ }^{1}$ Endocrinology, Department of Clinical Sciences, University of Rome 'La Sapienza', Rome, Italy; ${ }^{2}$ Department of \\ 'Clinica e Terapia Medica Applicata', University of Rome 'La Sapienza', Rome, Italy; ${ }^{3}$ Genome Centre, St \\ Bartholomew's and the Royal London, Queen Mary University of London, London, UK
}

Several genetic variants of peroxisome proliferator-activated receptor- $\gamma 2$ (PPAR- $\gamma 2$ ) have been identified, among which Pro12Ala, a missense mutation in exon 2, is highly prevalent in Caucasian populations. Up to now, conflicting results with regard to the association between this mutation and complex traits, such as obesity, insulin sensitivity and Type 2 diabetes, have been reported. We investigated the influence of the Pro12Ala polymorphism of PPAR- $\gamma 2$ on insulin sensitivity in a large Italian population sample, $n=1215$, in whom extensive clinical and biochemical analyses were performed. To estimate the insulin sensitivity status, the homeostasis model assessment of insulin resistance (HOMA-IR) was calculated; in the obese/ overweight subjects an oral glucose tolerance test (OGTT) was also performed and the Matsuda insulin sensitivity index (ISI) calculated. The insulin secretion index (homeostasis model assessment of percent $\beta$-cell function, HOMA- $\beta \%$ ) was utilized to evaluate $\beta$-cell function. The effect of the Pro12Ala polymorphism on quantitative variables was tested using multiple linear regression analysis. X12Ala (either Pro12Ala or Ala12Ala) genotype was associated with significantly lower fasting insulin levels compared to Pro/Pro ( $P=0.01$ after correction for multiple comparisons) in all subjects. Consistent with this finding, significantly lower HOMA-IR was observed in X12Ala carriers $(P=\mathbf{0 . 0 1 3}$ after correction for multiple comparisons) in all cohort. Moreover, no significant interaction effect was observed between body mass index and X12Ala polymorphism and between gender and X12Ala polymorphism in modulating insulin sensitivity. Our observations substantially extend previous findings and demonstrated that X12Ala variant is significantly associated with greater insulin sensitivity.

European Journal of Human Genetics (2004) 12, 1050-1054. doi:10.1038/sj.ejhg.5201283

Published online 15 September 2004

Keywords: homeostasis model assessment of insulin resistance; insulin sensitivity; peroxisome proliferatoractivated receptor- $\gamma 2$; obesity; body mass index

${ }^{*}$ Correspondence: Professor R Buzzetti, Endocrinology, Department of Clinical Sciences, University of Rome 'La Sapienza', Viale del Policlinico 155, Rome 00161, Italy. Tel.: + 3906 4478392; Fax: + 3906 4469190; E-mail: raffaella.buzzetti@uniroma1.it

Received 4 March 2004; revised 14 July 2004; accepted 15 July 2004
Introduction

Insulin resistance is a key factor in the development of Type 2 diabetes (T2DM) and multiple mechanisms contribute to its pathogenesis. Among these, the role of adipose tissue and obesity are of great significance. The metabolism and development of adipocytes is under complex regulation and recently the importance of the 
transcription factor peroxisome proliferator-activated receptor- $\gamma 2$ (PPAR- $\gamma 2)$ in these processes has been recognized.

PPAR- $\gamma$ are members of the nuclear hormone receptor family of transcription factors, known to control the expression of genes involved in the regulation of several metabolic processes. ${ }^{1,2}$ Alternative use of promoters and differential splicing of the PPAR- $\gamma$ gene results in three mRNA isoforms: PPAR $-\gamma 1,-\gamma 2$ and $-\gamma 3 .^{3}$ The PPAR- $\gamma 2$ is specific for adipose tissue; this characteristic makes it a likely candidate gene to be involved in the regulation of adipogenesis and lipid storage, insulin and glucose metabolism. Furthermore, this molecule controls the expression of secretory proteins such as leptin, adiponectin ${ }^{4}$ and tumor necrosis factor $\alpha,{ }^{5}$ which may act as modulators of insulin sensitivity in the skeletal muscles. ${ }^{1,6,7}$ These findings strongly suggest that PPAR- $\gamma 2$ may play a pivotal role in the whole body insulin sensitivity by influencing both adipocyte metabolism and insulin-stimulated process in nonadipose tissues.

Several genetic variants of PPAR- $\gamma 2$ have been identified, among which Pro12Ala, a missense mutation in exon $2,{ }^{8}$ is highly prevalent in Caucasian populations. ${ }^{2}$

Up to now, conflicting results with regard to the association between this mutation and complex traits, such as obesity, insulin sensitivity and T2DM, have been reported. Some of these proposed that the X12Ala (either Pro12Ala or Ala12Ala) genotype may be related to improved insulin sensitivity ${ }^{9,10}$ and protection from $\mathrm{T} 2 \mathrm{DM},{ }^{10,11}$ but this conclusion is still controversial.

The disparate findings may be partly attributed to insufficient power in some studies as well as to the heterogeneity of the population studied, in terms of clinical phenotypes. These factors highlight the need for much larger studies.

In view of these considerations, we evaluated the association of PPAR- $\gamma 2$ Pro12Ala polymorphism with measures of insulin sensitivity in a large population of individuals and assessed the relation of this genetic variant with the clinical and metabolic abnormalities of the insulin resistance state.

\section{Subjects and methods}

\section{Subjects}

We studied 1215 unrelated subjects who reside in the Lazio region (central Italy). Of these, normal-weight subjects (body mass index $(\mathrm{BMI})<25 ; n=400$ ) were randomly selected from a population of free-living individuals screened for coronary artery disease risk factors. Overweight/obese subjects (BMI $>25, n=815$ ) were consecutively recruited from the metabolic Day Hospital of the Department of Clinical Sciences of University 'La Sapienza' in Rome. Exclusion criteria were as follows: previous diagnosis of diabetes according to ADA criteria, ${ }^{12}$ chronic liver diseases and any treatment known to interfere with insulin sensitivity and metabolic syndrome-related parameters (hypolipemic drugs, insulin sensitizers, corticosteroid hormone, acetylsalicylic acid, $\beta$-blockers, oral contraceptives, thiazide diuretics, ACE inhibitors). All subjects gave their written informed consent to participate in the study after being informed of its nature. The study protocol was approved by the Ethical Committee of the University 'La Sapienza' in Rome.

In all subjects BMI, blood pressure, waist circumference (measured midway between the lowest rib margin and the iliac crest), hip circumference (measured over the great trocanthers) were measured as well as fasting glucose, insulin plasma levels and lipid profile (total, and HDL cholesterol and triglycerides). Furthermore, a 75-g oral glucose tolerance test (OGTT) was performed in all overweight/obese patients with glucose and insulin measurements at 30, 60, 90 and $120 \mathrm{~min}$.

\section{Methods}

Cholesterol and triglyceride concentrations were determined in the plasma by Technicon RA-1000 Autoanalyzer; HDL was measured after precipitation of ApoB-containing lipoproteins with phototungstic acid/ $\mathrm{MgCl}_{2}$. LDL-cholesterol was determined by the Friedewald formula. ${ }^{13}$ Glucose levels were calculated by the glucose oxidase method (Autoanalyzer, Beckman Coulter, USA). Plasma insulin was measured on frozen samples by radioimmunoassay (Adaltis insulin kit, Bologna, Italy), according to the manufacturing, with a limit of detection of $<2.0 \mu \mathrm{U} / \mathrm{ml}{ }^{14}$

Insulin resistance status was estimated according to homeostasis model assessment of insulin resistance (HOMA-IR) following the formula previously described: ${ }^{15}$ fasting insulin $(\mu \mathrm{U} / \mathrm{ml}) \times$ fasting glucose $(\mathrm{mmol} / \mathrm{l}) / 22.5$. Insulin sensitivity index (ISI) was calculated according to the formula $10000 / \sqrt{ }\left(\right.$ glucose $_{0} \times$ insulin $_{0} \times$ glucose $_{\text {mean }} \times$ insulin $_{\text {mean }) .}{ }^{16}$

To evaluate $\beta$-cell function, the insulin secretion index (homeostasis model assessment of percent $\beta$-cell function, HOMA- $\beta \%$ ) was computed as: $[20 \times$ fasting insulin $(\mathrm{mU} /$ 1)]/[(fasting glucose $(\mathrm{mmol} / \mathrm{l})-3.5]{ }^{15}$

In 100 subjects, insulin-stimulated glucose disposal measurements were performed by the euglycemic hyperinsulinemic clamp performed as previously described. ${ }^{17}$ The values obtained by the indexes approach correlated well with those obtained with the glucose clamp technique (data not shown).

\section{Genotype analysis}

The $200 \mathrm{bp}$ of sequence surrounding PPAR- $\gamma 2$ Pro12Ala was provided to Applied Biosystems to develop Taqman Allelic Discrimination (AD) Assays ${ }^{\mathbb{R}}$ using their assay by design platform (Foster City, CA, USA). Genotyping 
of the Pro12Ala AD was performed using primers forward 5'-TTATGGGTGAAACTCTGGGAGATT-3' and reverse 5'TGCAGACAGTGTATCAGTGAAGGA- $3^{\prime}$ and the Taqman MGB probes: Fam-TTCTGGGTCAATAGG; Vic-CTTTCTGC GTCAATAG. A measure of $4 \mu \mathrm{l}$ of a $10 \mathrm{ng} / \mu \mathrm{l}$ stock of DNA was dispensed into 384-well PCR plates using a Biomek FX robot (Fullerton, CA, USA) to which $6 \mu \mathrm{l}$ of a mix containing primers, probes and reagent mix (ABI, Foster City, CA, USA) were added according to the manufacturers' instructions. These were sealed with optical seals and incubated at $95^{\circ} \mathrm{C} 10 \mathrm{~min}$ followed by 40 cycles of $95^{\circ} \mathrm{C} 15 \mathrm{~s}$ and $60^{\circ} \mathrm{C} 1 \mathrm{~min}$ before analysis on a $7900 \mathrm{HT}$ plate reader (ABI, Foster City, CA, USA). Individual genotypes were determined using SDSv2.1 software (ABI, Foster City, CA, USA)

\section{Statistical analysis}

Statistical analysis was performed using SPSS statistical software, version 12 (SPSS, Illinois, USA). Genotypic and allelic distributions were compared using the $\chi^{2}$ test. The effect of the Pro12Ala polymorphism on the quantitative variables was investigated using multiple linear regression. We adjusted the crude effect of Pro12Ala polymorphism taking account of BMI, gender and age. The Pro12Ala polymorphism was introduced as a dichotomous variable in the analysis. In addition, the effects of the interaction between genotype and BMI and between genotype and gender were included in the model. Data for insulin, triglycerides, HOMA-IR, HOMA- $\beta$ and ISI were transformed using $\log _{10}$ to normalize their distributions.

\section{Results}

Observed genotype frequencies of the polymorphism were in agreement with the Hardy-Weinberg expectations. The frequency of Ala allele was 9.15\% in the whole cohort (Pro/ Pro: 83\%; Pro/Ala: 15.7\%; Ala/Ala: 1.3\%), similar to that reported in other Caucasian populations (Table 1). Impaired fasting glucose occurred in 60 (4.9\%) of all subjects; according to the results of OGTT, impaired glucose tolerance occurred in 134 (16.5\%) of overweight and obese subjects. The prevalence of women in menopausal status was $24 \%$ in our cohort.

The Pro12Ala variant was not associated with BMI at the $P=0.05$ level. No significant differences in age, gender, blood pressure, waist/hip ratio were noted between the X12Ala carriers and wild-type individuals (Table 1). We observed significantly lower fasting glucose levels in X12Ala carriers compared to Pro/Pro $(P=0.02)$ in all subjects. X12Ala genotypes were associated with significantly lower fasting insulin levels compared to Pro/Pro $(P=0.0008)$ in all cohort. Consistent with this finding, a significantly lower HOMA-IR was observed in X12Ala carriers $(P=0.001)$ compared to wild-type individuals in all subjects. Although not significant, the insulin secretion index HOMA- $\beta$ tended to be reduced in Ala carriers. The lipid profile did not show any significant differences in the groups analyzed (Table 2) in all individuals.

Among the overweight/obese subjects, Ala carriers had a significantly higher ISI index compared to wild-type individuals $(P=0.01)$. However, once we have corrected for multiple comparisons, using Bonferroni test, the fasting glucose $(P=0.26)$ and ISI OGTT $(P=0.13)$ significances

Table 1 Clinical features of subjects according to Pro12Ala PPAR- $\gamma 2$ genotype

\begin{tabular}{lccc}
\hline & Pro/Pro & Pro/Ala or Ala/Ala & P-value $^{\text {a }}$ \\
\hline$n$ (men/women) & $1008(303 / 705)$ & $207(63 / 144)$ & 0.75 \\
Age (years) & $42.6 \pm 13.6$ & $42.9 \pm 13.3$ & 0.34 \\
BMl (kg/m $\left.{ }^{2}\right)$ & $32.8 \pm 9$ & $32.4 \pm 10$ & 0.43 \\
Systolic BP (mmHg) & $128 \pm 19.84$ & $129 \pm 20.10$ & 0.78 \\
Diastolic BP (mmHg) & $81.3 \pm 11.53$ & $0.5 \pm \pm 0.09$ & 0.12 \\
Waist/hip & $0.84 \pm 0.08$ & $0.83 \pm 0$ \\
\hline
\end{tabular}

${ }^{\mathrm{a}}$ Data are given as means and SD. All comparisons are adjusted for age, BMI, gender, PPAR- $\gamma 2 \times$ BMI interaction and PPAR- $\gamma 2 \times$ gender interaction.

Table 2 Biochemical parameters of subjects according to Pro12Ala PPAR- $\gamma 2$ genotype

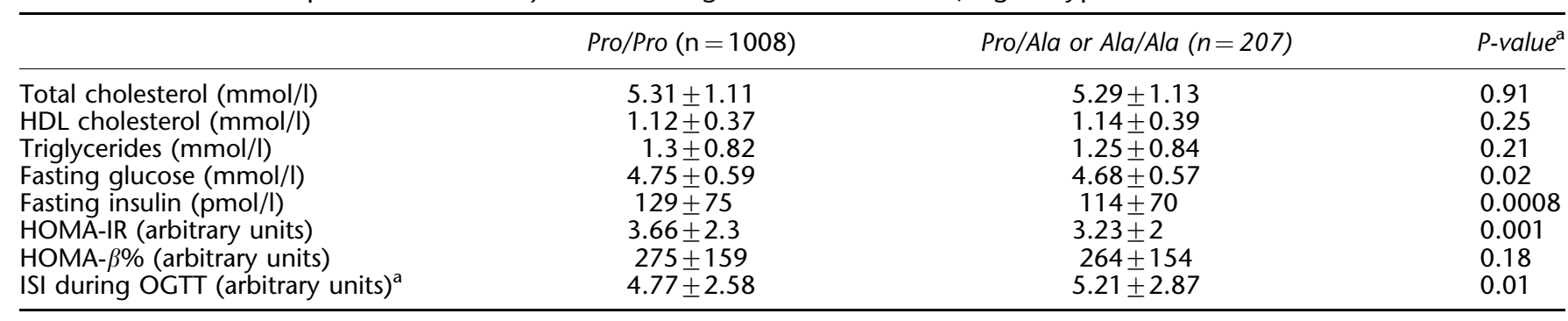

${ }^{\mathrm{a}}$ Data are given as means and SD. All comparisons are adjusted for age, BMI, gender, PPAR $-\gamma 2 \times$ BMI interaction and PPAR- $\gamma 2 \times$ gender interaction. 
were no longer statistically significant at the 5\% level. The significance levels of fasting insulin and HOMA-IR remained significant after correction for multiple comparisons ( $P=0.01$ and 0.013 , respectively). No significant interaction effect was observed between BMI and X12Ala polymorphism (HOMA-IR P-value interaction 0.51 , fasting insulin $P$-value interaction 0.55 and fasting glucose $P$-value interaction 0.66 ) and between gender and X12Ala polymorphism in modulating insulin sensitivity (HOMA-IR $P$-value interaction 0.71, fasting insulin $P$-value interaction 0.68 and fasting glucose $P$-value interaction 0.75).

\section{Discussion}

In the present study, we have demonstrated that the common variant of PPAR- $\gamma 2$, X12Ala, present in Italian population with an allele frequency of $9.15 \%$, is significantly associated with higher insulin sensitivity. In particular, X12Ala carriers showed significantly lower insulin levels and HOMA-IR and in subjects within the overweight/obese group a significantly higher ISI index compared to wild-type individuals (Table 2). We did not observe any significant difference between the two genotypes in the lipid profile; however, we observed a significantly lower fasting glucose level in all cohort. It is likely that improved insulin sensitivity could be responsible for the significant decrease in fasting glucose levels. To our knowledge, a significantly higher ISI index in X12Ala carriers compared to wild-type individuals has not been reported previously. The first evidence for an association between the X12Ala polymorphism in PPAR- $\gamma 2$ and increased insulin sensitivity was reported by Debb et al. ${ }^{9}$ The Authors found a significant increase of the Ala allele in individuals with normal glucose tolerance compared to T2DM patients and a significantly greater insulin sensitivity in nondiabetic Ala carriers. Only one ${ }^{18}$ of the five subsequent studies ${ }^{19-23}$ reproduced the original findings; however, a meta-analysis, including the previous five and Scandinavian parent-offspring trios, demonstrated that the Pro12Ala polymorphism significantly decreases the risk of T2DM. ${ }^{11}$ Greater insulin sensitivity was observed in normo glucose-tolerant Swedish 70-year-old men carrying the Ala allele; ${ }^{24}$ in subgroups of obese subjects, the increase in insulin sensitivity was also more pronounced. $^{25}$ Stumvoll and co-workers demonstrated that the Ala allele of PPAR- $\gamma 2$ becomes particularly advantageous against the background of an additional, possibly disadvantageous genetic polymorphism such as the G972R variant of IRS- $1 .{ }^{26}$

The picture, however, is still unclear as reported in a Swedish middle-aged man in whom a significantly higher body mass with predominance of abdominal distribution of adipose tissue was observed along with a marked impairment in estimated insulin sensitivity in homozygotes Ala carriers. ${ }^{10}$ Moreover, as reported by Frederiksen et $a l^{27}$ in our study we did not observe an interaction between the variant and BMI on the examined variables.

The conflicting findings may be partly attributed to insufficient power in some studies, the heterogeneity of the population studied in terms of clinical phenotype (lean, obese) and type of study (population based, clinical based).

Altshuler et al $^{11}$ pointed out that despite the population impact of common risk alleles such as 12 Ala, their contribution will be impossible to discover by linkage analysis (requiring a genome scan of millions of sib pairs) and therefore association studies are required. Even so, studies with modest sample sizes might fail to detect true associations. Therefore, the dissection of common risk alleles involved in multifactorial diseases should entail association studies performed on large population samples as in the present study, which involves a number of subjects substantially higher compared to the majority of studies performed so far on this matter.

One key question that deserves an answer is related to the contribution of Pro12Ala polymorphism in regulating insulin secretion. Normal weight Ala carriers showed a decreased second-phase insulin secretion following elevation of serum-free fatty acids ${ }^{28}$ and a significant lower insulin secretion was observed in T2DM patients carrying the Ala allele compared to Pro12Pro subjects. ${ }^{29}$ In our large population study, despite a tendency for a decrease in HOMA- $\beta \%$, there was no significant correlation between insulin secretion and Ala allele.

Two independent studies have observed a reduced transcriptional activity of PPAR- $\gamma 2$ as a result of Pro-Ala exchange. ${ }^{9,30}$ Our and previous observations suggest that the X12Ala polymorphism improves insulin sensitivity. These findings are in line with observations in heterozygous PPAR $\gamma$-deficient transgenic mice, which have reduced transcriptional activity of PPAR $\gamma$ and are protected from high-fat diet obesity and insulin resistance. ${ }^{31,32}$ However, it is still currently unclear by which mechanisms a reduced transcriptional activity of PPAR $\gamma$ (both in humans and in animal models) could influence insulin sensitivity. In our study, we did not observe any significant difference in lipid profile, waist/hip ratio, blood pressure and BMI between the X12Ala carriers and wild-type individuals. As it is well recognized that some adipocytokines (resistin, TNFa, adiponectin) are under the transcriptional control of PPAR- $\gamma 2$, we could speculate that any of them could mediate the effect of Pro12Ala on insulin sensitivity.

In conclusion, we observed by using several measurements of insulin sensitivity, including fasting insulin, HOMA-IR and ISI, a protective effect of the X12Ala polymorphism of PPAR- $\gamma 2$ on insulin resistance; future 
studies investigating the functional effect of this polymorphism will help to clarify the exact role of X12Ala variant.

\section{Acknowledgements}

We thank all the patients for their participation. We thank Silvia Di Cola for their excellent technical assistance. This work has been supported in part by a grant from the Ministry of Health (ICS 030.6/ RFO0-49) and in part from The Ministry of The University and Scientific Research (MIUR grant n: 2003061834-004; 2003).

\section{References}

1 Spiegelman BM: PPAR-gamma: adipogenic regulator and thiazolidinedione receptor. Diabetes 1998; 47: 507-514.

2 Stumvoll M, Haring H: The peroxisome proliferator-activated receptor-gamma2 Pro12Ala polymorphism. Diabetes 2002; 51: 2341-2347.

3 Fajas L, Debril MB, Auwerx J: PPAR gamma: an essential role in metabolic control. Nutr Metab Cardiovasc Dis 2001; 11: 64-69.

4 Weyer C, Funahashi T, Tanaka S et al: Hypoadiponectinemia in obesity and type 2 diabetes: close association with insulin resistance and hyperinsulinemia. J Clin Endocrinol Metab 2001; 86: 1930-1935.

5 Kroder G, Bossenmaier B, Kellerer $\mathrm{M}$ et al: Tumor necrosis factoralpha- and hyperglycemia-induced insulin resistance. Evidence for different mechanisms and different effects on insulin signaling. J Clin Invest 1996; 97: 1471-1477.

6 Gervois P, Torra IP, Fruchart JC, Staels B: Regulation of lipid and lipoprotein metabolism by PPAR activators. Clin Chem Lab Med 2000; 38: 3-11.

7 Olefsky JM: Treatment of insulin resistance with peroxisome proliferator-activated receptor gamma agonists. J Clin Invest 2000; 106: $467-472$.

8 Yen CJ, Beamer BA, Negri C et al: Molecular scanning of the human peroxisome proliferator activated receptor gamma (hPPAR gamma) gene in diabetic Caucasians: identification of a Pro12Ala PPAR gamma 2 missense mutation. Biochem Biophys Res Commun 1997; 241: 270-274.

9 Deeb SS, Fajas L, Nemoto $M$ et al: A Pro12Ala substitution in PPARgamma2 associated with decreased receptor activity, lower body mass index and improved insulin sensitivity. Nat Genet 1998; 20: 284-287.

10 Rosmond R, Chagnon M, Bouchard C: The Pro12Ala PPARgamma2 gene missense mutation is associated with obesity and insulin resistance in Swedish middle-aged men. Diabetes Metab Res Rev 2003; 19: 159-163.

11 Altshuler D, Hirschhorn JN, Klannemark M et al: The common PPARgamma Pro12Ala polymorphism is associated with decreased risk of type 2 diabetes. Nat Genet 2000; 26: 76-80.

12 Expert Committee on the Diagnosis and Classification of Diabetes Mellitus: Report of the expert committee on the diagnosis and classification of diabetes mellitus. Diabetes Care 2000; 1 (Suppl 23): S4-S19.

13 Friedewald WT, Levy RJ, Fredrickson DS: Estimation of the concentration of low-density-lipoprotein cholesterol in plasma without use of the preparative ultracentrifuge. Clin Chem 1972; 18: $499-502$.

14 Gorden P, Lesniak MA, Hendricks CM, Roth J: 'Big' growth hormone components from human plasma: decreased reactivity demonstrated by radioreceptor assay. Science 1973; 182: 829-831.

15 Matthews DR, Hosker JP, Rudenski AS, Naylor BA, Treacher DF, Turner RC: Homeostasis model assessment: insulin resistance and beta-cell function from fasting plasma glucose and insulin concentrations in man. Diabetologia 1985; 28: 412-419.
16 Matsuda M, DeFronzo RA: Insulin sensitivity indices obtained from oral glucose tolerance testing: comparison with the euglycemic insulin clamp. Diabetes Care 1999; 22: 1462-1470.

17 Iacobellis G, Ribaudo MC, Zappaterreno A et al: Relationship of insulin sensitivity and left ventricular mass in uncomplicated obesity. Obes Res 2003; 11: 518-524.

18 Hara K, Okada T, Tobe $\mathrm{K}$ et al: The Pro12Ala polymorphism in PPAR gamma2 may confer resistance to type 2 diabetes. Biochem Biophys Res Commun 2000; 271: 212-216.

19 Globerman H, Zauberman Y, Makarov T et al: Analysis of the peroxisome proliferator activated receptor gamma (PPARgamma) gene in HAIRAN syndrome with obesity. Clin Endocrinol 2000; 52: $479-485$.

20 Meirhaeghe A, Fajas L, Helbecque $\mathrm{N}$ et al: A genetic polymorphism of the peroxisome proliferator-activated receptor gamma gene influences plasma leptin levels in obese humans. Hum Mol Genet 1998; 7: 435-440.

21 Clement K, Hercberg S, Passinge B et al: The Pro115Gln and Pro12Ala PPAR gamma gene mutations in obesity and type 2 diabetes. Int J Obes Relat Metab Disord 2000; 24: 391-393.

22 Mancini FP, Vaccaro O, Sabatino L et al: Pro12Ala substitution in the peroxisome proliferator-activated receptor-gamma2 is not associated with type 2 diabetes. Diabetes 1999; 48: $1466-1468$.

23 Ringel J, Engeli S, Distler A, Sharma AM: Pro12Ala missense mutation of the peroxisome proliferator activated receptor gamma and diabetes mellitus. Biochem Biophys Res Commun 1999; 254: 450-453.

24 Ek J, Andersen G, Urhammer SA et al: Mutation analysis of peroxisome proliferator-activated receptor-gamma coactivator-1 (PGC-1) and relationships of identified amino acid polymorphisms to Type II diabetes mellitus. Diabetologia 2001; 44: $2220-2226$

25 Koch M, Rett K, Maerker E et al: The PPARgamma2 amino acid polymorphism Pro 12 Ala is prevalent in offspring of Type II diabetic patients and is associated to increased insulin sensitivity in a subgroup of obese subjects. Diabetologia 1999; 42: $758-762$.

26 Stumvoll M, Stefan N, Fritsche A et al: Interaction effect between common polymorphisms in PPARgamma2 (Pro12Ala) and insulin receptor substrate 1 (Gly972Arg) on insulin sensitivity. J Mol Med 2001; 80: 33-38.

27 Frederiksen L, Brodbaek K, Fenger M, Jorgensen T, Borch-Johnsen K, Madsbad S, Urhammer SA: Comment: studies of the Pro12Ala polymorphism of the PPAR-gamma gene in the Danish MONICA cohort: homozygosity of the Ala allele confers a decreased risk of the insulin resistance symdrome. J Clin Endocrinol Metab 2002; 87: 3989-3992.

28 Stefan N, Fritsche A, Haring H, Stumvoll M: Effect of experimental elevation of free fatty acids on insulin secretion and insulin sensitivity in healthy carriers of the Pro12Ala polymorphism of the peroxisome proliferator-activated receptor-gamma2 gene. Diabetes 2001; 50: 1143-1148.

29 Mori H, Ikegami H, Kawaguchi $\mathrm{Y}$ et al: The Pro12 ->Ala substitution in PPAR-gamma is associated with resistance to development of diabetes in the general population: possible involvement in impairment of insulin secretion in individuals with type 2 diabetes. Diabetes 2001; 50: 891-894.

30 Masugi J, Tamori Y, Mori H, Koike T, Kasuga M: Inhibitory effect of a proline-to-alanine substitution at codon 12 of peroxisome proliferator-activated receptor-gamma 2 on thiazolidinedioneinduced adipogenesis. Biochem Biophys Res Commun 2000; 268: $178-182$.

31 Kubota N, Terauchi Y, Miki $\mathrm{H}$ et al: PPAR gamma mediates highfat diet-induced adipocyte hypertrophy and insulin resistance. Mol Cell 1999; 4: 597-609.

32 Miles PD, Barak Y, He W, Evans RM, Olefsky JM: Improved insulin-sensitivity in mice heterozygous for PPAR-gamma deficiency. J Clin Invest 2000; 105: 287-292. 